\title{
Cost-Effectiveness of Pembrolizumab for the Adjuvant Treatment of Melanoma Patients with Lymph Node Involvement Who Have Undergone Complete Resection in Argentina
}

\author{
Victoria Wurcel (D) - Emilie Scherrer - Raquel Aguiar-Ibanez • \\ Juan Ignacio Altuna · Fernando Carabajal · Shrishti Jain • \\ Gargi Baluni
}

Received: December 17, 2020 / Accepted: January 23, 2021 / Published online: February 23, 2021

(c) The Author(s) 2021

\section{ABSTRACT}

Introduction: The KEYNOTE-054 trial demonstrated that adjuvant pembrolizumab improves recurrence-free survival in completely resected stage III melanoma versus watchful waiting (hazard ratio $[\mathrm{HR}]=0.57 ; 98.4 \%$ confidence interval [CI], 0.43-0.74). We evaluated the costeffectiveness of pembrolizumab in Argentina, where watchful waiting is still widely used among these patients despite the high risk of recurrence with surgery alone.

Methods: A four-health state model was used (recurrence-free, locoregional recurrence [LR], distant metastases [DM], death). Lifetime medical costs to payers (72.08 Argentine pesos [AR $\$=1.00$ U.S. dollar [USD]) and outcomes (3\% annual discount) were assessed, together with incremental cost-effectiveness ratios (ICERs). First and $\mathrm{LR} \rightarrow \mathrm{DM}$ recurrences were modeled using KEYNOTE-054 and real-world data, respectively. No benefits of adjuvant treatment were assumed post-progression. Pre-

V. Wurcel $(\bowtie)$. J. I. Altuna · F. Carabajal MSD Argentina, Buenos Aires, Argentina e-mail: victoria.wurcel@merck.com

E. Scherrer · R. Aguiar-Ibanez

Merck \& Co., Inc, Kenilworth, NJ, USA

S. Jain · G. Baluni

Complete HEOR Solutions, CHEORS, North Wales,

PA, USA
DM and post-DM mortality was based on KEYNOTE-054 and on a network meta-analysis of advanced treatments expected in each arm, respectively. Utilities were derived from KEYNOTE-054 Euro-QoL data using an Argentinian algorithm, and from the literature. Public exfactory drug prices were used.

Results: Patients in the pembrolizumab and the watchful waiting arms accrued 8.78 and 5.83 quality-adjusted life-years (QALYs), 9.91 and 6.98 life-years, and costs of AR $\$ 12,698,595$ $(176,174$ USD) and AR $\$ 11,967,717(166,034$ USD), respectively. The proportion of life-years accrued that were recurrence-free was $80.8 \%$ and $56.9 \%$ in the pembrolizumab and the watchful waiting arms, respectively. Pembrolizumab patients gained 2.94 life-years and 2.96 QALYs versus watchful waiting; the ICER per QALY was AR\$247,094 (3428 USD). Recurrence rates and advanced melanoma treatments were the key drivers of the ICER. At a threshold of AR $\$ 1,445,325$ (29,935 USD) per QALY, pembrolizumab had an $83.5 \%$ probability of being cost-effective versus watchful waiting.

Conclusions: Adjuvant pembrolizumab after complete resection of melanoma with node involvement is highly cost-effective relative to watchful waiting in Argentina, across disease stage subgroups and BRAF mutational status. This strongly supports its coverage and reimbursement across the entire health system. 
Keywords: Adjuvant treatment; Argentina; Cost-effectiveness; Latin America; Melanoma; Pembrolizumab

\section{Key Summary Points}

Why carry out this study?

Adjuvant treatment with pembrolizumab is effective in patients with stage III melanoma who have undergone complete resection.

In Argentina, watchful waiting is still widely used for these patients despite the high risk of recurrence with surgery alone.

We assessed whether pembrolizumab for adjuvant treatment of melanoma is costeffective in Argentina in comparison to watchful waiting.

\section{What was learned from the study?}

Adjuvant pembrolizumab after complete resection of melanoma with lymph node involvement is highly cost-effective compared with watchful waiting in Argentina.

The results support the inclusion of pembrolizumab in the compulsory medical plan for all eligible patients across the entire Argentinian health care system.

\section{DIGITAL FEATURES}

This article is published with digital features, including a summary slide, to facilitate understanding of the article. To view digital features for this article go to https://doi.org/10.6084/ m9.figshare.13615415.

\section{INTRODUCTION}

Melanoma accounts for the highest share of deaths due to skin neoplasms [1]. The American Cancer Society reports that the 5-year relative survival rate for melanoma patients in the United States is $98 \%$ for localized melanomas, $64 \%$ for locoregional melanoma with lymph node involvement, and $23 \%$ for distant metastatic disease [2]. The frequency of the disease has been steadily increasing in recent decades and is currently growing by $1.2 \%$ annually [2]. Worldwide, 287,723 cases were diagnosed in 2018, with higher rates among males than females [3]. In South America, the incidence of about 3 cases per 100,000 [4] is low compared to rates of between 19.4 and 41.8 cases per 100,000 seen in Oceania, North America, and Europe [5]. In spite of this fact, melanoma is responsible for 32.5 disability-adjusted life-years (DALYs) per 100,000 population in southern Latin America [6]. In Argentina, 1703 men and women were diagnosed in 2018, reflecting an incidence of 3.8 cases per 100,000 [7].

Most melanoma patients are diagnosed with early-stage disease, but recurrence is seen in many cases (15-40\%) despite the original favorable prognosis [8-10]. There are also a considerable number of patients first diagnosed in advanced stage III or IV disease. [2] For resectable stage III disease in Argentina, the standard of care is excision with safety margins based on the presence and depth of invasion plus lymphadenectomy if regional lymph nodes are involved, followed by observation alone or with systemic adjuvant therapies targeting residual micrometastatic disease. Despite the high risk of disease recurrence in patients with stage III melanoma managed with surgery alone, a watchful waiting strategy in completely resected patients continues to be the most common treatment approach in Argentina.

The first adjuvant treatments for melanoma were high-dose interferon- $\alpha 2 b$ and peginterferon- $\alpha 2 b$, which showed modest efficacy but considerable toxicity [11-13] These regimens have since been displaced by the introduction of immune checkpoint inhibitors, anti-programmed death-1 (PD-1) monoclonal antibodies, and targeted drugs active in BRAF-positive mutated melanoma [14]. Pembrolizumab is a novel high-affinity monoclonal antibody that blocks the activity of the PD-1 receptor reactivating the tumor-specific cytotoxic T-lymphocyte response [15]. A randomized, controlled, 
double-blind, two-arm trial (KEYNOTE-054) showed that adjuvant treatment with pembrolizumab significantly improves recurrencefree survival in this patient population as compared to placebo (hazard ratio $[\mathrm{HR}]=0.57$; $98.4 \%$ confidence interval [CI], 0.43-0.74; $p<0.0001$ ) [16]. Subsequently, the National Administration of Drugs, Foods, and Medical Devices [Administración Nacional de Medicamentos, Alimentos y Tecnología Médica (ANMAT)] approved pembrolizumab for adjuvant therapy in this indication in Argentina.

To support la Comisión Nacional de Evaluación de Tecnologías de Salud (CONETEC)—-the Argentinian national health technology assessment (HTA) body-in making decisions about financing cancer treatments, we evaluated the cost-effectiveness of adjuvant pembrolizumab relative to watchful waiting following complete resection of melanoma with lymph node involvement, taking a health care system perspective.

\section{METHODS}

\section{Population}

The overall target population for the economic evaluation is aligned with the patient population enrolled in the KEYNOTE-054 trial and the Argentinian label: melanoma patients with lymph node involvement who have undergone complete resection $[16,17]$. Subgroup analyses were conducted according to BRAF mutation status and melanoma staging and classification of disease defined according to the American Joint Committee on Cancer (AJCC) Staging Manual (7th edition): stage IIIA, stage IIIB, stage IIIC with 1-3 positive lymph nodes, and stage IIIC with $\geq 4$ positive lymph nodes [18].

\section{Model Structure and Analysis}

The Markov cohort model, developed in Excel, consists of four mutually exclusive health states: recurrence-free, locoregional recurrence, distant metastases, and death (Fig. 1).
Completely resected stage III melanoma patients enter the model in the recurrence-free state and then may experience locoregional recurrence, distant metastases, or death. Death can occur pre- or post-recurrence (Fig. 1). The analysis was undertaken on a 46-year time horizon to reflect the lifetime of an average patient entering the model at age 54 as in KEYNOTE-054. The cost-effectiveness analysis took the health care system perspective reflecting CONETEC composition. The direct medical care costs, expressed in 2020 Argentine pesos (AR\$; 72.08 AR\$ = 1 US dollar [USD] [19]), and health outcomes in terms of life-years and quality-adjusted life-years (QALYs) were discounted by $3 \%$ annually, in alignment with World Health Organization Choosing Interventions that are Cost-Effective (WHOCHOICE) recommendations on economic evaluation of health technologies [20].

The distribution of individuals across health states was used in conjunction with statespecific costs and health utilities to estimate total expected lifetime costs and QALYs with pembrolizumab adjuvant treatment as compared to a watchful waiting strategy. Incremental cost-effectiveness ratios (ICERs) were calculated in terms of cost per QALY gained and per life-year gained. To evaluate the robustness of the incremental cost-utility ratio, a series of one-way sensitivity analyses were conducted by modifying key model settings and varying model parameters deterministically across a range of plausible values, together with a 1000 -iteration probabilistic sensitivity analysis

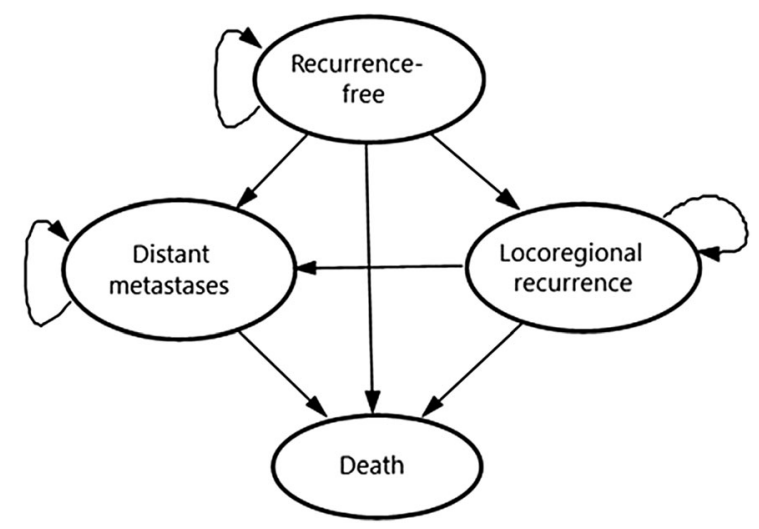

Fig. 1 Model schematic 
Table 1 Model inputs

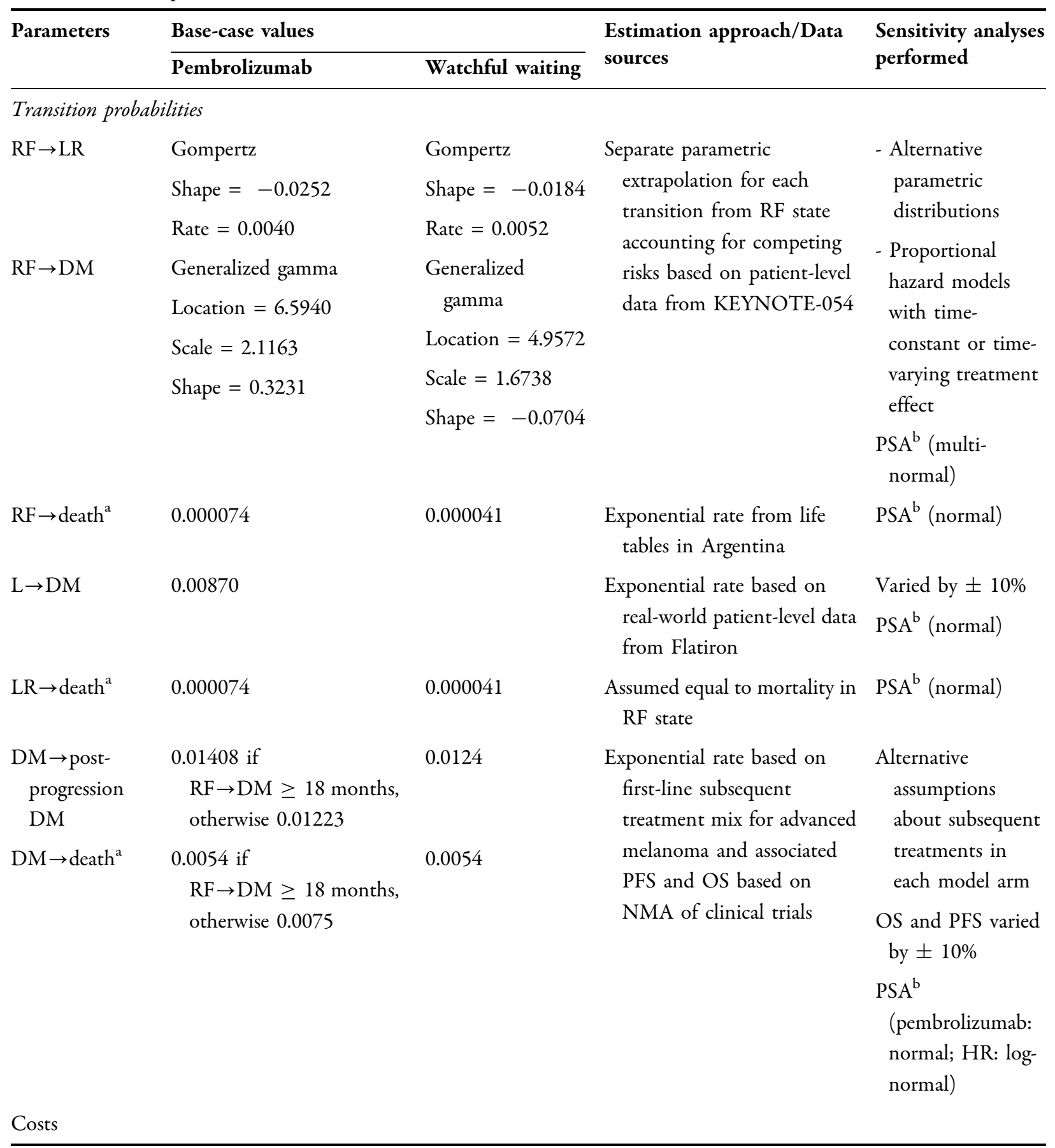


Table 1 continued

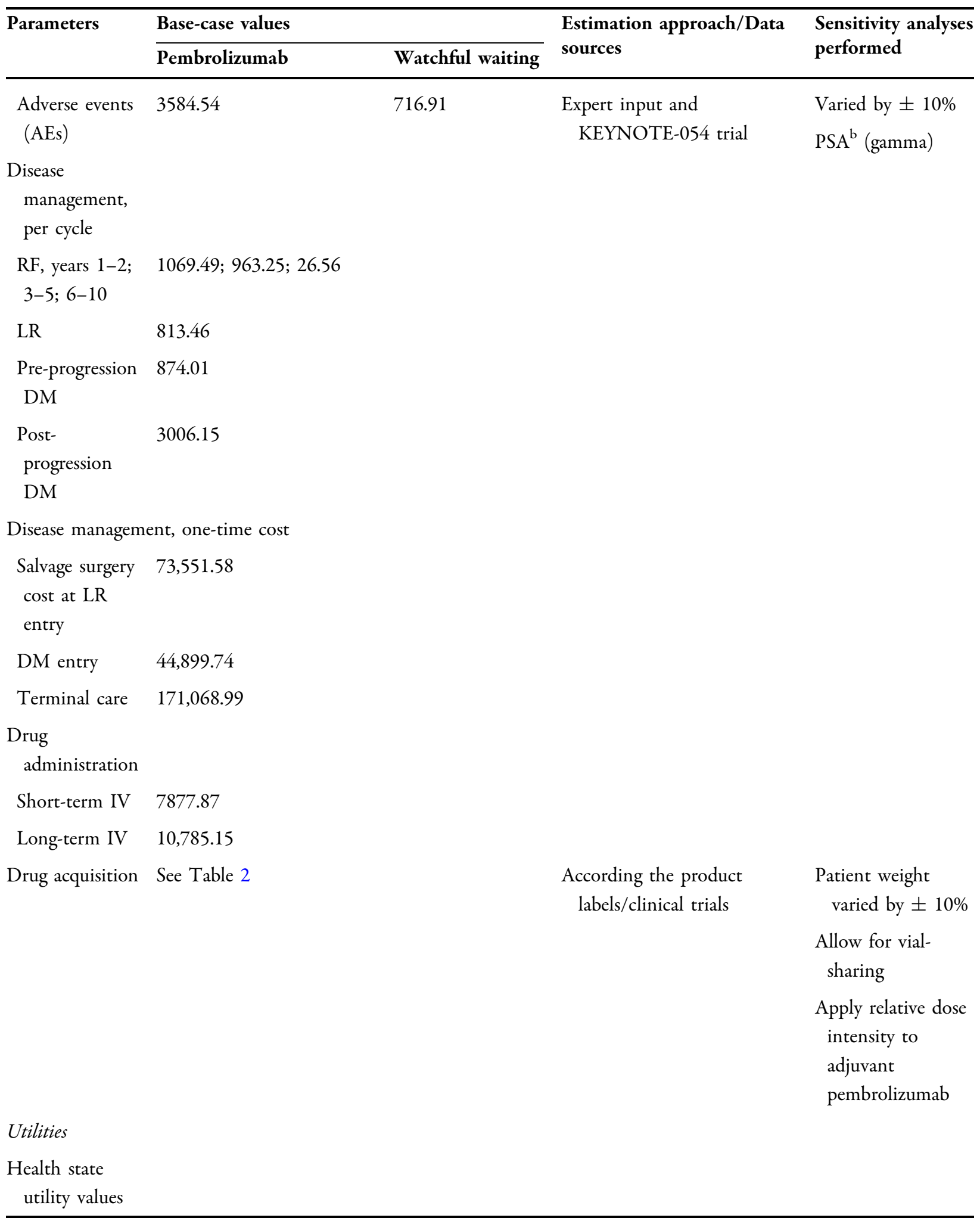


Table 1 continued

\begin{tabular}{|c|c|c|c|c|}
\hline \multirow[t]{2}{*}{$\overline{\text { Parameters }}$} & \multicolumn{2}{|l|}{ Base-case values } & \multirow{2}{*}{$\begin{array}{l}\text { Estimation approach/Data } \\
\text { sources }\end{array}$} & \multirow{2}{*}{$\begin{array}{l}\text { Sensitivity analyses } \\
\text { performed }\end{array}$} \\
\hline & Pembrolizumab & Watchful waiting & & \\
\hline $\begin{array}{l}\text { RF without } \\
\text { toxicity }\end{array}$ & 0.923 & & KEYNOTE-054 trial & $\begin{array}{l}\text { Varied by } \pm 10 \% \\
\text { Alternative source }\end{array}$ \\
\hline LR & 0.860 & & & for post- \\
\hline $\begin{array}{l}\text { Pre-progression } \\
\text { DM }\end{array}$ & 0.837 & & & $\begin{array}{l}\text { progression DM } \\
\text { state }\end{array}$ \\
\hline $\begin{array}{l}\text { Post- } \\
\text { progression } \\
\text { DM }\end{array}$ & 0.590 & & Beusterien et al. & $\begin{array}{l}\text { PSA }^{\mathrm{b}} \text { (health state: } \\
\text { beta; AE-related } \\
\text { distutilities: }\end{array}$ \\
\hline $\begin{array}{l}\text { Disutility from } \\
\text { adverse events }\end{array}$ & -0.082 & & KEYNOTE-054 trial & normal) \\
\hline
\end{tabular}

$D M$ distant metastases, $H R$ hazard ratio, $L R$ locoregional recurrence, $N M A$ network meta-analysis, $O S$ overall survival, $P F S$ progression-free survival, $P S A$ probabilistic sensitivity analysis, $R F$ recurrence-free

a Transition probabilities to death constrained to be at least as high as all-cause mortality from the national life tables

b The variability in the selected distributions was based on standard errors or variance-covariance from original data sources. For costs, the standard errors were assumed to be equal to $20 \%$ of the base-case values

(PSA) varying parameter values simultaneously. Standard errors or variance-covariance matrices for the selected probability distributions were based on original data sources, where available, and otherwise were set at $20 \%$ of mean values (Table 1).

\section{INPUTS}

\section{Transition Probabilities}

A parametric approach was used to estimate transition probabilities between multiple states in which competing failures are treated as censoring events to account for concurrent risks [21-24]. All transitions probabilities were estimated using $\mathrm{R}$ software.

Locoregional and distant metastatic recurrence from the recurrence-free state was modeled separately for each arm using patient-level data from KEYNOTE-054 (Fig. 2). In line with the approach recommended by the National Institute for Health and Care Excellence (NICE)
Decision Support Unit (DSU) for partitioned survival models [25], the same distribution types were selected for both treatment arms, and the least-squares mean goodness of fit between predicted and observed values was optimized in conjunction with visual inspection as described by Williams et al. [22] Six candidate distributions were considered to model recurrence: exponential, Weibull, Gompertz, log-logistic, log-normal, and generalized gamma. Distributions were therefore selected from the 36 possible combinations for locoregional recurrence and distant metastatic recurrence; in the base case, the distributions were Gompertz and generalized gamma, respectively. The selected combination ranked first for watchful waiting and fifth for pembrolizumab according to statistical goodness of fit. Alternative distributions were tested in scenario analyses (Table 1).

After a recurrence occurred, no ongoing benefit from active adjuvant treatment was assumed for subsequent transitions (Fig. 2). In the absence of mature post-recurrence data 

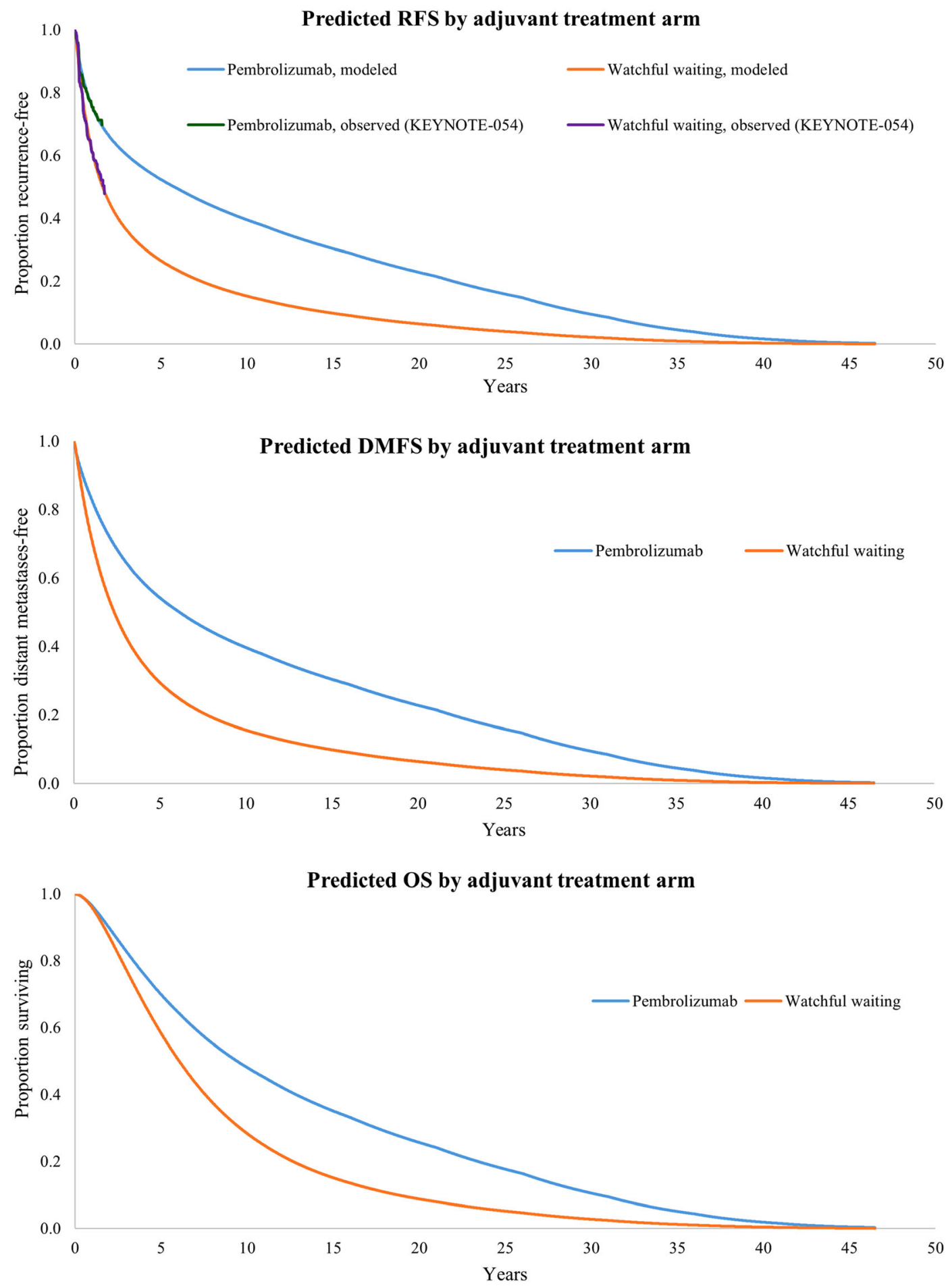

Fig. 2 Predicted long-term outcomes under base-case parametric distribution assumptions in the overall patient population. a Recurrence-free survival (RFS). b Distant metastasis-free survival (DMFS). c Overall survival (OS)

from KEYNOTE-054 interim analysis, an exponential model for the transition from locoregional recurrence to distant metastatic state was fitted using real-world US data from 
Table 2 Drug regimens and unit costs

\begin{tabular}{|c|c|c|}
\hline Drugs & Dosing schedule ${ }^{a}$ & $\begin{array}{l}\text { Unit cost, AR\$ (strength, } \\
\text { mg) }\end{array}$ \\
\hline Pembrolizumab & $200 \mathrm{mg}$ IV Q3W (as adjuvant, up to 18 cycles) & $194,860.81(100)$ \\
\hline Ipilimumab & $3 \mathrm{mg} / \mathrm{kg} \mathrm{IV} \mathrm{(with} \mathrm{nivolumab,} 1 \mathrm{mg} / \mathrm{kg}$ ) Q3W, up to 4 doses & $200,289.03(50)$ \\
\hline \multirow[t]{2}{*}{ Nivolumab } & $240 \mathrm{mg}$ IV Q2W as monotherapy & $40,697.08(40)$ \\
\hline & $\begin{array}{l}3 \mathrm{mg} / \mathrm{kg} \text { IV Q3W followed by ipilimumab (up to } 4 \text { doses), } 240 \mathrm{mg} \text { Q2W } \\
\text { thereafter }\end{array}$ & $101,745.77(100)$ \\
\hline Dabrafenib & Dabrafenib $150 \mathrm{mg}$ b.i.d orally in combination with trametinib & $3078.11(75)$ \\
\hline Trametinib & Trametinib 2 mg q.d. orally in combination with dabrafenib & $6474.94(2)$ \\
\hline Dacarbazine & $1800 \mathrm{mg} / \mathrm{m}^{2} \mathrm{IV}, \mathrm{Q} 3 \mathrm{~W}$ & $2053.40(200)$ \\
\hline
\end{tabular}

the Flatiron Health electronic medical record database; [26] 147 stage III melanoma patients who had undergone complete resection and experienced locoregional recurrence were included. As no death was observed from the locoregional recurrence state during follow-up, the risk of death from the locoregional recurrence and the recurrence-free states was assumed to be equal and was estimated from KEYNOTE-054 and constrained to being no lower than in the general population of similar age and gender [27]. Considering the small number of deaths from these health states, an exponential distribution was used.

From the distant metastatic state, the probability of death was estimated for each arm based on the expected mix of first-line treatments for advanced melanoma approved in Argentina (pembrolizumab, ipilimumab, nivolumab, nivolumab + ipilimumab, and dabrafenib + trametinib), for which the efficacy in terms of progression-free survival and overall survival was assessed based on trials in the advanced melanoma setting (Fig. 2). For pembrolizumab, these distributions were fitted using patient-level data from those receiving pembrolizumab $10 \mathrm{mg} / \mathrm{kg}$ every 3 weeks (Q3W arm; first-line subgroup) in the KEYNOTE-006 trial among ipilimumab-naïve patients with unresectable/advanced melanoma [28]. For other first-line treatments, HRs for progressionfree and overall survival versus pembrolizumab were estimated through an NMA of trials [28-35]. Overall survival in the distant metastatic state was calculated separately for each arm as a weighted average of expected survival with the different first-line treatments for advanced melanoma. At any time, the risk of death could not be lower than in the general population of similar age and gender (Table 1). The rate of disease progression in the distant metastatic state was used for the calculation of QALYs, disease management costs, and subsequent treatment costs in the advanced melanoma setting and was estimated in a similar manner. To reflect real-world practice, market shares in the watchful waiting arm were obtained from Argentina-specific market research and expert panel inputs. In the pembrolizumab arm, patients' eligibility for subsequent PD-1 inhibitors was expected to depend on whether metastases occurred early or late. Specifically, when recurrence-free patients were diagnosed with distant metastatic disease and completed a course of treatment at least 18 months following the initiation of an adjuvant treatment, patients were re-challenged with pembrolizumab; otherwise, it was assumed 
that patients could not be treated with a PD-1 inhibitor, and they were distributed to other subsequent treatments (details given in the costs section).

\section{Adverse Events}

The model considered grade 3 or higher adverse events (AEs) with a frequency of at least 5\% (all grades) in either of the KEYNOTE-054 trial arms. Medical costs and health disutilities were applied to AEs of grade 3 or higher. Diarrhea of grade 2 was also factored in given the considerable economic consequences associated with its management.

\section{Health Utilities}

Health utilities were assessed through an Argentinian algorithm [36] applied to EuroQol five-dimension three-level (EQ-5D-3L) KEYNOTE-054 data after pooling both arms. Health state utility values and disutilities associated with AEs were derived from a mixed-regression model with patient-level random effects. Given the limited distant metastatic follow-up in KEYNOTE-054 interim analysis, for the basecase scenario the post-progression distant metastatic state utility was informed by published literature in advanced melanoma (Table 1) [37]. The utility value in that state was estimated as the weighted average of the preand post-progression values based on the expected distant metastatic progression-free and overall survival considering the market shares of first-line treatments for advanced melanoma. Mean durations of AEs were collected from KEYNOTE-054 to calculate AE-related QALY decrements, which were applied as a one-time decrement in the first model cycle.

\section{Costs}

Direct medical care costs from the perspective of the health care system in Argentina included the costs for adjuvant treatment drug acquisition and administration, AEs, disease management, first- and second-line subsequent treatments in the advanced melanoma setting, and terminal care.

All medical resource mean unit costs were obtained from different Argentinian institutions selected to ensure a representative sample from public, private, and social insurance health care settings [38]. The private costs were sourced from three health maintenance organizations in Argentina. Their frequency of use was assessed based on the input gathered from local guidelines and validated during an expert input forum. Data from the KEYNOTE-054 trial informed health care resource utilization related to managing AE episodes (Table 1). Medical cost in the recurrence-free state included outpatient services such as physician office visits and radiologic assessments and were assumed to decrease over time based on guideline recommendations [39]. Following locoregional recurrence, a one-time cost for salvage surgery was applied based on the proportion of patients who received lymphadenectomy, skin lesion resection, in-transit metastases resection, or other surgery after locoregional recurrence in KEYNOTE-054, and the respective health care costs for these procedures [17]. Following distant metastatic recurrence, a one-time cost was applied based on medical resources associated with first-line treatment initiation [40]. Subsequently, weekly medical costs were applied, as well as a one-time terminal care cost for patients who died after experiencing a distant metastatic recurrence [41].

Table 2 describes drug ex-factory prices based on Argentina's public list price database [42] together with the treatment regimen dosing schedules determined according to Argentina's product labeling and randomized clinical trial protocols. The dosing schedule of pembrolizumab in the adjuvant setting is $200 \mathrm{mg}$ Q3W for up to 18 cycles (1 year). The actual time on adjuvant treatment was applied according to the KEYNOTE-054 Kaplan-Meier treatment duration curve, without the need for extrapolation. For melanoma treatment administered once patients develop distant metastasis, the market shares were informed by market research and expert inputs [43]. It was assumed that recurrence-free patients who developed distant metastasis at least 18 months 
after initiation of adjuvant treatment would be re-challenged with pembrolizumab. For the others, $60 \%$ received ipilimumab as first-line advanced treatment and $40 \%$ received dabrafenib + trametinib. In the watchful waiting arm, pembrolizumab, ipilimumab, nivolumab, nivolumab + ipilimumab, and dabrafenib + trametinib were the first-line treatment options in the following respective proportions: $27.5 \%, 8.0 \%, 29.5 \%, 30.0 \%$, and $5.0 \%$. For each treatment regimen, the exponential rate of progression-free survival failure assessed from the NMA was used to approximate the treatment discontinuation rate, up to the label-recommended maximum duration where applicable. The cost of second-line therapies for advanced melanoma were also accounted for, although no impact of these regimens on progression-free or overall survival was factored in. Based on expert input panel information, it was assumed that all patients from the watchful waiting arm and 90\% of those from the adjuvant pembrolizumab arm would receive an active second-line treatment in the advanced setting. The assumptions about the distribution of the second-line therapies depended on the adjuvant arm and on whether pembrolizumab patients were re-challenged after progression to the distant metastatic state. Patients who received adjuvant treatment with pembrolizumab and who were re-challenged in the metastatic setting received ipilimumab $(30.0 \%)$, dabrafenib + trametinib $(10.0 \%)$ or dacarbazine $(50 \%)$ as a second-line advanced treatment. Among patients in the pembrolizumab arm who were not re-challenged in the advanced melanoma setting, 30.0\%, 45.0\%, and $\quad 15 \%$ received ipilimumab, dabrafenib + trametinib, and dacarbazine, respectively. In the watchful waiting arm, patients received either pembrolizumab (24.5\%), ipilimumab (7.5\%), nivolumab (17.5\%), nivolumab + ipilimumab (21.5\%), dabrafenib + trametinib $(16.9 \%)$, or dacarbazine (12.0\%) as a second-line advanced treatment. The duration of each second-line therapy was based on a mean of 21 weeks on treatment but capped to any maximum as described in Table 2. Cost calculation of weightbased dosages assumed no vial-sharing and were based on the weight of an average Argentine patient provided by experts $(75 \mathrm{~kg}$ for males and $65 \mathrm{~kg}$ for females) [43]. The administration cost of short-time complex parenteral chemotherapy was applicable to monotherapy regimes, while the cost of long-term complex parenteral chemotherapy was applied to combination therapy [38]. Orally administered drugs were assumed to require no administration costs.

\section{COMPLIANCE WITH ETHICS GUIDELINES}

This article is based on previously conducted studies and does not contain any studies with human participants or animals performed by any of the authors.

\section{RESULTS}

\section{BASE CASE}

The discounted base-case results for the overall population over a 46-year lifetime horizon are shown in Table 3. Total costs were AR\$12,698,595 (176,174 USD) for patients receiving pembrolizumab adjuvant treatment as compared to AR $\$ 11,967,717$ (166,034 USD) for patients in the watchful waiting arm. The difference was largely driven by the costs of adjuvant treatment in the pembrolizumab arm and of subsequent treatments which were higher in the watchful waiting arm. Disease management costs were also lower in the pembrolizumab arm (AR\$383,448; 5320 USD) than in the watchful waiting arm (AR\$468,905; 6505 USD). This was also true for terminal care costs, which were lower for pembrolizumab (AR\$91,663; 1272 USD) compared to watchful waiting (AR\$126,381; 1753 USD). With adjuvant pembrolizumab, patients lived a total of 9.91 discounted life-years and accrued 8.78 QALYs as compared to 6.98 life-years and 5.83 QALYs in the watchful waiting arm. The proportion of total life-years that were accrued within the recurrence-free state was $80.8 \%$ in the 
Table 3 Base-case results

\begin{tabular}{|c|c|c|c|}
\hline Costs and outcomes & Watchful waiting & Pembrolizumab & Incremental \\
\hline Costs, AR\$ & $11,967,717$ & $12,698,595$ & 730,878 \\
\hline Adjuvant treatment & 0 & $5,569,375$ & $5,569,375$ \\
\hline Drug acquisition & 0 & $5,459,026$ & $5,459,026$ \\
\hline Drug administration & 0 & 110,349 & 110,349 \\
\hline Subsequent treatment & $11,371,121$ & $6,650,525$ & $-4,720,596$ \\
\hline Drug acquisition & $11,129,387$ & $6,582,875$ & $-4,546,512$ \\
\hline Drug administration & 241,734 & 67,650 & $-174,084$ \\
\hline Adverse event & 1310 & 3585 & 2275 \\
\hline Disease management & 468,905 & 383,448 & $-85,457$ \\
\hline Terminal care & 126,381 & 91,663 & $-34,718$ \\
\hline QALYs & 5.83 & 8.78 & 2.96 \\
\hline Recurrence-free & 3.66 & 7.40 & 3.74 \\
\hline Locoregional recurrence & 0.33 & 0.23 & -0.10 \\
\hline Distant metastases & 1.83 & 1.16 & -0.67 \\
\hline AE-related disutility & -0.0004 & -0.0006 & 0.00 \\
\hline Life-years & 6.98 & 9.91 & 2.94 \\
\hline Recurrence-free & 3.97 & 8.01 & 4.04 \\
\hline Locoregional recurrence & 0.39 & 0.26 & -0.13 \\
\hline Distant metastases & 2.62 & 1.64 & -0.98 \\
\hline \multicolumn{4}{|c|}{ Cost-effectiveness ratio, AR\$ } \\
\hline Per life-year & & & 247,094 \\
\hline Per QALY & & & 248,879 \\
\hline
\end{tabular}

QALY quality-adjusted life-year. 72.08 AR $\$=1$ USD [19]

pembrolizumab arm versus $56.9 \%$ in the watchful waiting arm. Patients treated with adjuvant pembrolizumab gained 2.94 life-years and 2.96 QALYs over watchful waiting, resulting in ICERs of AR $\$ 247,094$ (3428 USD) per QALY gained and AR $\$ 248,879$ (3453 USD) per life-year gained.

\section{Subgroup Analyses}

In subgroup analyses, the results ranged from pembrolizumab being dominant in stage IIIB (more effective, less costly) to pembrolizumab having an ICER per QALY gained of AR\$1,196,840 (16,604 USD) over watchful waiting in the stage IIIC (4+ lymph nodes) subgroup. The ICER per QALY gained according to the BRAF mutation status was AR $\$ 303,870$ (4216 USD) and AR\$560,253 (7773 USD) among 
Table 4 Subgroup analysis for cost-effectiveness of adjuvant pembrolizumab vs. watchful waiting in Argentina

\begin{tabular}{|c|c|c|c|c|c|c|c|c|}
\hline \multirow{2}{*}{ Subgroups and comparators } & \multirow{2}{*}{ Costs (AR\$) } & \multirow{2}{*}{ QALYs } & \multirow{2}{*}{ LYs } & \multicolumn{3}{|l|}{ Incremental } & \multicolumn{2}{|l|}{ ICER } \\
\hline & & & & Costs (AR\$) & QALYs & LYs & $\begin{array}{l}\text { AR\$/ } \\
\text { QALY }\end{array}$ & AR\$/LY \\
\hline \multicolumn{9}{|l|}{ All patients } \\
\hline Watchful waiting & $11,967,717$ & 5.83 & 6.98 & - & - & - & - & - \\
\hline Pembrolizumab & $12,698,595$ & 8.78 & 9.91 & 730,878 & 2.95 & 2.93 & 247,094 & 248,879 \\
\hline \multicolumn{9}{|l|}{$B R A F(-)$} \\
\hline Watchful waiting & $12,459,719$ & 5.00 & 6.15 & - & - & - & - & - \\
\hline Pembrolizumab & $13,816,570$ & 7.42 & 8.46 & $1,356,851$ & 2.42 & 2.31 & 560,253 & 587,012 \\
\hline \multicolumn{9}{|l|}{$B R A F(+)$} \\
\hline Watchful waiting & $12,321,979$ & 5.95 & 7.17 & - & - & - & - & - \\
\hline Pembrolizumab & $13,149,238$ & 8.67 & 9.83 & 827,259 & 2.72 & 2.65 & 303,870 & 311,666 \\
\hline \multicolumn{9}{|l|}{ Stage IIIA } \\
\hline Watchful waiting & $11,299,058$ & 7.06 & 8.33 & - & - & - & - & - \\
\hline Pembrolizumab & $11,850,120$ & 11.65 & 12.91 & 551,062 & 4.59 & 4.58 & 119,927 & 120,209 \\
\hline \multicolumn{9}{|l|}{ Stage IIIB } \\
\hline Watchful waiting & $12,306,278$ & 5.62 & 6.78 & - & - & - & - & - \\
\hline Pembrolizumab & $11,855,955$ & 9.88 & 11.04 & $-450,322$ & 4.26 & 4.26 & Dominant & Dominant \\
\hline \multicolumn{9}{|l|}{ Stage IIIC (1-3 lymph nodes) } \\
\hline Watchful waiting & $12,735,742$ & 4.84 & 5.95 & - & - & - & - & - \\
\hline Pembrolizumab & $12,982,753$ & 7.93 & 9.01 & 247,012 & 3.08 & 3.05 & 80,152 & 80,906 \\
\hline \multicolumn{9}{|l|}{ Stage IIIC (4+ lymph nodes) } \\
\hline Watchful waiting & $13,257,040$ & 4.39 & 5.50 & - & - & - & - & - \\
\hline Pembrolizumab & $14,756,942$ & 5.64 & 6.62 & $1,499,902$ & 1.25 & 1.13 & $1,196,840$ & $1,332,402$ \\
\hline
\end{tabular}

ICER incremental cost-effectiveness ratio, $L Y$ life-year, $Q A L Y$ quality-adjusted life-year 72.08 AR\$ $=1$ USD [19]

positive and negative patients, respectively (Table 4).

ranged from being a dominant strategy when transitions from the recurrence-free state to the distant metastatic state were modeled based on

\section{Sensitivity Analyses}

Figure 3 shows the sensitivity analyses with the greatest effect on the incremental cost per QALY gained associated with pembrolizumab versus watchful waiting. Across the scenarios tested in sensitivity analyses, adjuvant pembrolizumab a Gompertz distribution as opposed to a generalized gamma, to having an ICER per QALY gained of AR $\$ 983,505$ (13,645 USD) over watchful waiting when parametric models with a time-constant treatment effect were used for the cause-specific hazards of transitions from the recurrence-free state. The incremental cost 


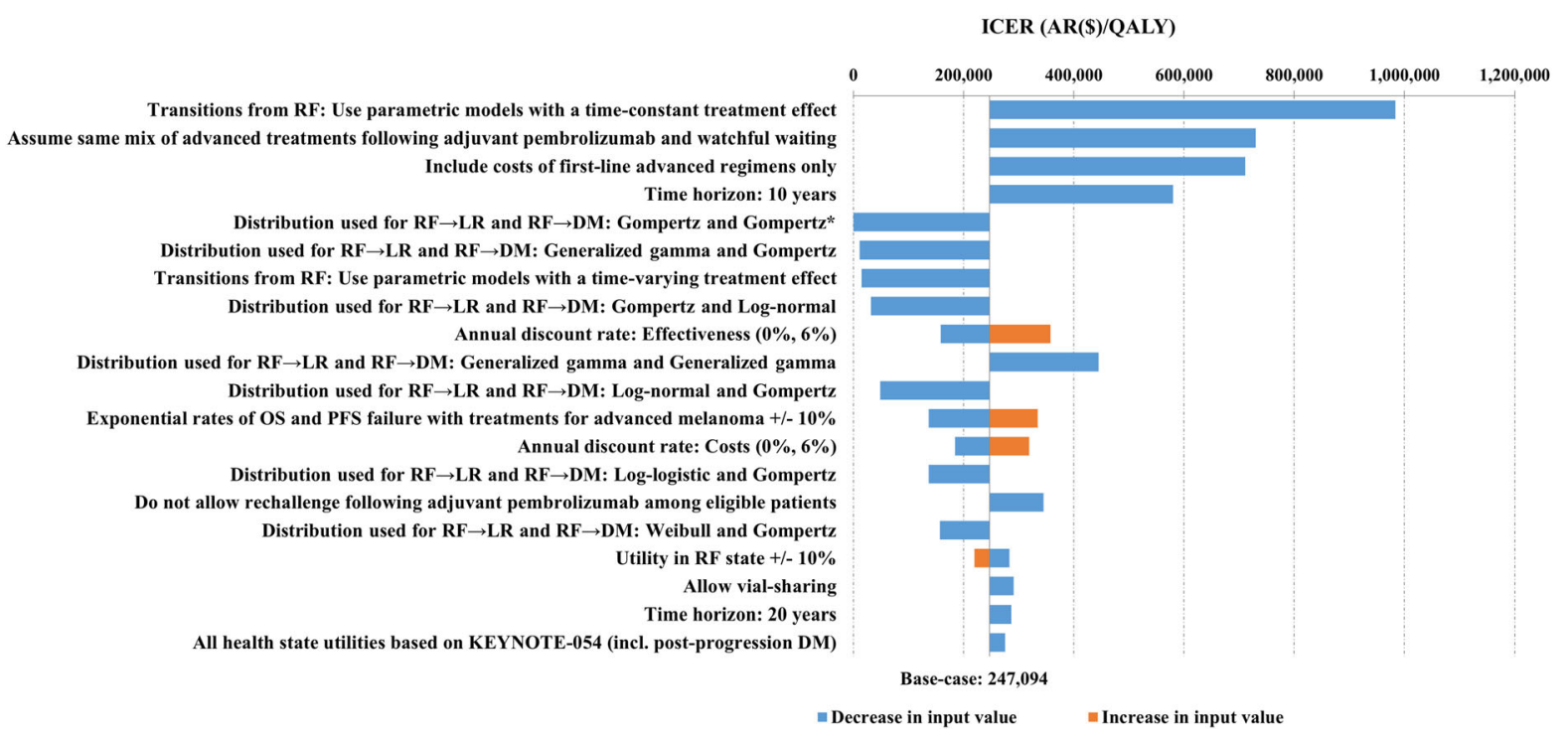

Fig. 3 Tornado diagram for the incremental cost-effectiveness ratio of adjuvant pembrolizumab vs. watchful waiting. $D M$ distant metastases, $H R$ hazard ratio, $L R$ analyses in which pembrolizumab is dominant over the comparator locoregional recurrence, $O S$ overall survival, PFS progression-free survival, $R F$ recurrence-free. ${ }^{*}$ Indicates sensitivity

\section{Incremental cost and effectiveness plane: Pembrolizumab vs. watchful waiting}

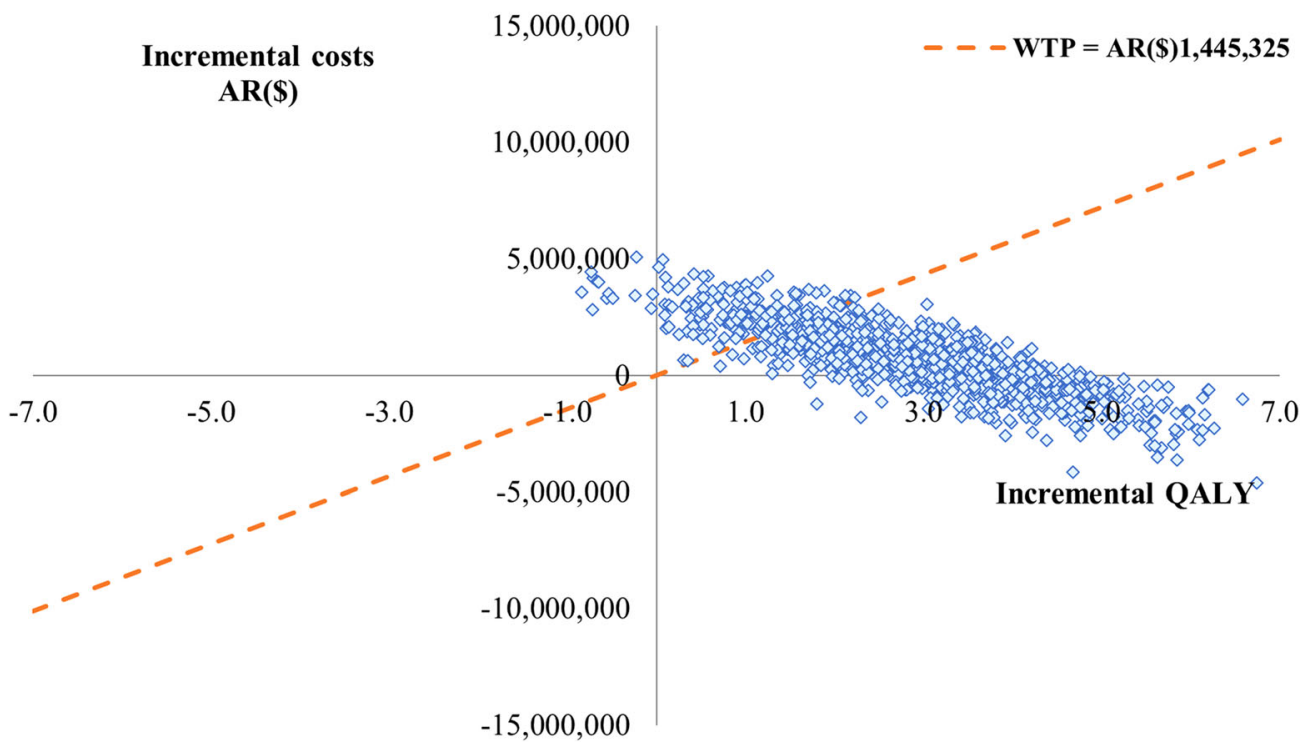

Fig. 4 Scatterplot of incremental costs and effectiveness for pembrolizumab vs. watchful waiting across 1000 iterations of the probabilistic sensitivity analysis. PSA

probabilistic sensitivity analysis, $W T P$ willingness to pay, QALY quality-adjusted life-year 
per QALY gained was AR $\$ 345,177$ (4789 USD) when patients treated with pembrolizumab in the adjuvant setting could not be re-challenged, AR \$710,940 (9863 USD) when the second-line treatments in the advanced melanoma setting were excluded, and AR $\$ 729,866$ (10,126 USD) when the market shares of subsequent treatments were set equal in both arms. The result was not substantially higher when using a short 10-year time horizon, with an ICER of AR \$580,067 (8048 USD) per QALY gained, nor were the results sensitive to other model features, such as discount rate for costs and outcomes, patient weight and vial-sharing assumptions, drug administration and other non-drug medical care costs, locoregional recurrence and distant metastatic state health utilities and AE-related disutilities, or mortality from the locoregional recurrence state.

Figure 4 presents a scatterplot of the simulated incremental cost and QALY pair for the comparison of adjuvant pembrolizumab treatment with watchful waiting. Across the 1000 iterations of the PSA, the average incremental cost was AR $\$ 778,340$ (10,798 USD) and the average incremental QALY gain was 2.85 for pembrolizumab versus watchful waiting, for a probabilistic ICER per QALY gained of AR $\$ 272,726$ (3784 USD). Based on a willingness-to-pay threshold of AR $\$ 1,445,325(29,935$ USD) per QALY, equivalent to three times the per capita gross domestic product (GDP) in Argentina in 2019 [44], adjuvant pembrolizumab had an $83.5 \%$ probability of being cost-effective versus watchful waiting.

\section{DISCUSSION}

Despite a large proportion of resected high-risk stage III melanoma patients developing disease recurrence when managed with surgery alone [8-10], and novel adjuvant therapies such as pembrolizumab becoming available in Argentina, equal access is not ensured across the health care system. Timely access becomes difficult especially for melanoma patients covered by public health insurance. To highlight that adjuvant pembrolizumab treatment in melanoma is a valuable and sustainable option for
Argentina's health care system, we assessed the cost-effectiveness of pembrolizumab compared with watchful waiting. We found pembrolizumab to be highly cost-effective as an adjuvant treatment following complete resection of stage III melanoma with lymph node involvement in comparison to watchful waiting, and as such, a good value proposition for Argentina's health care system, deserving coverage and reimbursement. Over a lifetime horizon, adjuvant pembrolizumab was projected to yield substantial survival gains and QALY improvements. By delaying recurrence, adjuvant pembrolizumab is expected to result in approximately 3 additional life-years and also to provide about 3 additional QALYs per treated patient. These gains came at reasonable additional costs, because investing in pembrolizumab adjuvant treatment resulted in savings in total costs of subsequent treatments, disease managements and terminal care in the adjuvant pembrolizumab group. This reflects the lower incidence of recurrence and lower expected share of more expensive subsequent combination treatments and health care resource utilization in the adjuvant pembrolizumab group compared with watchful waiting. According to the WHO-CHOICE project [45], interventions with an ICER below three times the per capita GDP for each QALY gained should be considered cost-effective, and those costing less than the per capita GDP should be viewed as highly cost-effective. Under base-case assumptions, pembrolizumab was associated with an ICER of AR \$247,094 (3428 USD) per QALY gained, well below the per capita GDP in Argentina of AR $\$ 481,775$ (9978 USD) as compared to watchful waiting. At a willingness-to-pay threshold of three times the per capita GDP in Argentina (AR \$1,445,325; 29,935 USD) for each QALY gained, pembrolizumab was cost-effective versus watchful waiting in the vast majority of scenarios (83.5\%) as well as across a range of plausible input values and alternative modeling assumptions with robust ICERs. Recurrence rates and subsequent advanced melanoma treatments were the key drivers of the ICER, with pembrolizumab ranging from being economically dominant (more effective, less costly) to remaining cost-effective 
with an ICER of AR $\$ 983,505$ (13,645 USD) per QALY gained, when parametric models for disease recurrence assumed a time-constant treatment effect which resulted in a worse fit of the KEYNOTE-054 trial data as compared to the base-case method. Pembrolizumab also remained cost-effective regardless of BRAF-mutation status and in all disease stage subgroups, especially in stage IIIB patients, where adjuvant pembrolizumab was dominant over watchful waiting.

These results are in accordance with those seen in the US setting, where adjuvant pembrolizumab was found to be highly cost-effective versus watchful waiting $(15,009$ USD/QALY gained) for patients with high-risk stage III melanoma [46]. The slightly lower number of life-years gained with adjuvant pembrolizumab in Argentina (2.94) versus the US (3.39) reflects higher mortality rates in the Argentinian population as compared with those seen in North America, and different distribution of subsequent advanced melanoma treatments in each country.

This economic evaluation is based on a wellestablished Markov modeling approach that has been commonly used for submission of oncology drugs to internationally recognized HTA organizations. We focused on the within-trial direct comparison of costs and effectiveness between pembrolizumab and watchful waiting. Risks of recurrence were modeled parametrically in each arm using patient-level data from KEYNOTE-054, the phase 3 trial that directly compared pembrolizumab and watchful waiting. In the absence of mature post-recurrence data from the trial, post-recurrence transition probabilities were estimated using real-world data and network-meta analysis of clinical trials in the advanced melanoma setting. The selection of parametric functions to model transitions was based on goodness of fit with the observed data, and clinical plausibility of long-term extrapolations assessed using external data and expert opinion, in concordance with recommended practice [25]. The use of recurrence-free survival as a surrogate end-point to extrapolate long-term overall survival within the model is supported by the natural history of resected high-risk melanoma and by a recent meta- analysis of randomized controlled trials of adjuvant interferon treatment of resected highrisk melanoma [47] showing a high degree of correspondence between the HR for recurrencefree and overall survival. The 5 -year data from the EORTC 18071 trial similarly showed a relative reduction in recurrence or death with ipilimumab versus placebo (HR: 0.76 and 0.72 , respectively). In addition, the 5-year overall survival in the placebo arm of the EORTC 18071 trial (54.4\%) was similar to the predicted overall survival in the watchful waiting arm of the model $(56.8 \%)$, which further supports the plausibility of our extrapolations [48, 49]. Additionally, given the 1-year maximum duration of adjuvant pembrolizumab, the time on treatment in the adjuvant pembrolizumab arm was precisely estimated based on the observed Kaplan-Meier data from KEYNOTE-054, without the need for extrapolation. Moreover, the model was inclusive of the mix of subsequent treatments reflecting the clinical practice in Argentina for advanced melanoma in each arm, with their efficacy in terms of progression-free and overall survival assessed from a network meta-analysis. This analysis also included quality-of-life estimates adjusted to reflect utilities from Argentinian patients, which contributes to the external validity and applicability of the results.

As with any cost-effectiveness analysis, there are limitations to the economic model that need to be acknowledged. First, we focused on direct medical care costs; the inclusion of societal dimensions such as productivity loss could further improve the cost-effectiveness profile of the adjuvant treatment. Second, after the model was completed, new KEYNOTE-054 data were published confirming and showing an even slightly higher recurrence risk reduction in the 3 -year assessment for pembrolizumab in the overall population (HR 0.56 ; 95\% CI 0.47-0.68). This further supports the benefit of pembrolizumab in the adjuvant setting in relation to recurrence-free survival. Additionally, because the KEYNOTE-054 trial interim data cutoff used for this analysis were too immature to inform the post-recurrence transition probabilities, the model assumed that adjuvant pembrolizumab would have no ongoing 
therapeutic benefit once disease recurred. Advanced melanoma and overall survival predictions are therefore subject to greater uncertainty and should be validated against longterm efficacy data from the KEYNOTE-054 trial as they become available. Nevertheless, we have tried to limit this uncertainty by a thorough sensitivity analysis. There is also some uncertainty with respect to treatments used in the advanced melanoma settings, for instance, in terms of eligibility of pembrolizumab for subsequent immunotherapies. Modifying the assumptions about the mix of subsequent advanced melanoma treatments to be received in each arm, however, did not alter the conclusion about the cost-effectiveness of adjuvant pembrolizumab.

\section{CONCLUSIONS}

Adjuvant pembrolizumab after complete resection of melanoma with lymph node involvement is highly cost-effective in Argentina. The results are sustained across all analyzed patient subgroups including BRAF mutational status, and strongly support the coverage and reimbursement of pembrolizumab for all eligible patients across the entire Argentinian health care system.

\section{ACKNOWLEDGEMENTS}

Funding. Sponsorship for this study and the journals's Rapid Service Fee were funded by Merck Sharp \& Dohme Corp., a subsidiary of Merck \& Co., Inc., Kenilworth, NJ, USA.

Medical Writing, Editorial, and Other Assistance. The authors would like to thank Martin Sénécal (Complete Health Economics Outcomes and Research Solutions) for providing editorial and overall assistance for the writeup and development of this article, as well as Pablo Andre (MSD Argentina), Hernán Barugel (MSD Argentina), Roberto Gomez (MSD Argentina), Karina Mendez (MSD Argentina), Raúl Soler (MSD Argentina), Katia Wendt (MSD
Panama), Cristina Okuyama (Merck \& Co., Inc., Kenilworth, NJ, USA), Alfredo Caparros (MSD Argentina), Miguel Korte (Merck \& Co., Inc., Kenilworth, NJ USA), Jaime Enrique Ruiz (MSD Colombia), Homero Monsanto (Merck \& Co., Inc., Kenilworth, NJ, USA), José Salvador Velazco (MSD Mexico), and Fernando Zuelgaray (MSD Argentina) for their editorial and scientific peer review, Maggie Hodgson (Merck \& Co., Inc., Kenilworth, NJ, USA) for providing scientific publication advice, and Ana Canchola Mendez (Merck \& Co., Inc., Kenilworth, NJ, USA), Camille Simpson (Merck \& Co., Inc., Kenilworth, NJ, USA), Tyrone Brewer (Merck \& Co., Inc., Kenilworth, NJ, USA) for their project implementation and funding assistance. MSD provided funding to support the write-up and the development of this article.

Authorship. All named authors meet the International Committee of Medical Journal Editors (ICMJE) criteria for authorship for this article, take responsibility for the integrity of the work as a whole, and have given their approval for this version to be published.

Disclosures. Victoria Wurcel, Juan Ignacio Altuna, and Fernando Carabajal are employees of MSD Argentina, Buenos Aires, Argentina. Emilie Scherrer and Raquel Aguiar-Ibanez are employees of Merck Sharp \& Dohme Corp., a subsidiary of Merck \& Co., Inc., Kenilworth, NJ, USA. Shrishti Jain and Gargi Baluni are employees of CHEORS, North Wales, PA, USA.

Compliance with Ethics Guidelines. This article is based on previously conducted studies and does not contain any studies with human participants or animals performed by any of the authors. The authors had permission to access the databases needed to perform this study.

Data Availability. The datasets generated during and/or analyzed during the current study are not publicly available due to their proprietary nature. Further information about the data and conditions for access can be obtained from the corresponding author. 
Open Access. This article is licensed under a Creative Commons Attribution-NonCommercial 4.0 International License, which permits any non-commercial use, sharing, adaptation, distribution and reproduction in any medium or format, as long as you give appropriate credit to the original author(s) and the source, provide a link to the Creative Commons licence, and indicate if changes were made. The images or other third party material in this article are included in the article's Creative Commons licence, unless indicated otherwise in a credit line to the material. If material is not included in the article's Creative Commons licence and your intended use is not permitted by statutory regulation or exceeds the permitted use, you will need to obtain permission directly from the copyright holder. To view a copy of this licence, visit http://creativecommons.org/licenses/by$\mathrm{nc} / 4.0 /$.

\section{REFERENCES}

1. National Cancer Institute. Cancer Facts \& Figures 2020. CA Cancer J Clin 2020:1-76.

2. American Cancer Society. Melanoma of the skin cancer stat facts. https://www.cancer.org/es/cancer/ cancer-de-piel-tipo-melanoma/detecciondiagnostico-clasificacion-por-etapas/tasas-desupervivencia-para-el-cancer-de-piel-de-tipomelanoma-por-etapas.html. Accessed 20 Nov 2020.

3. International Agency for Research on Cancer Globocan 2018. Melanoma of skin - fact sheet [Internet]. Available from: https://gco.iarc.fr/. Accessed 20 Nov 2020.

4. Loria D, Abriata G, Santoro F, Latorre C (2020) Cutaneous melanoma in Argentina: an analysis of its characteristics and regional differences. Ecancermedical Sci. https://doi.org/10.3332/ ecancer.2020.1017.

5. International Agency for Research on Cancer Globocan (2018) Cancer incidence in five continents. https://ci5.iarc.fr/Default.aspx. Accessed 20 Nov 2020.

6. Karimkhani C, Green AC, Nijsten T, et al. (2017) The global burden of melanoma: results from the Global Burden of Disease Study 2015. Br J Dermatol. https://doi.org/10.1111/bjd.15510.
7. International Agency for Research on Cancer Globocan 2018. Argentina - fact sheet. Available from: https:/gco.iarc.fr/today/data/factsheets/ populations/32-argentina-fact-sheets.pdf.

8. Jang S, Poretta T, Bhagnani T, et al. (2020) Correction to: Real-world recurrence rates and economic burden in patients with resected early-stage melanoma. Dermatol Ther (Heidelb) 10(5):985-999. https://doi.org/10.1007/s13555-020-00404-9.

9. Salama AKS, de Rosa N, Scheri RP et al (2013) Hazard-rate analysis and patterns of recurrence in early stage melanoma: moving towards a rationally designed surveillance strategy. PLoS One. https:// doi.org/10.1371/journal.pone.0057665.

10. Gershenwald JE, Scolyer RA, Hess KR, et al. (2017) Melanoma staging: evidence-based changes in the American Joint Committee on Cancer eighth edition cancer staging manual. CA Cancer J Clin 67(6): 472-492. https://doi.org/10.3322/caac.21409.

11. Sondak VK, Wolfe JA (1997) Adjuvant therapy for melanoma. Curr Opin Oncol 9(2):189-204. https:// doi.org/10.1097/00001622-199703000-00015.

12. Kirkwood JM, Ibrahim JG, Sondak VK, et al. (2002) Interferon alfa-2a for melanoma metastases. Lancet 359(9310):978-979. https://doi.org/10.1016/S01406736(02)08001-7.

13. Herndon TM, Demko SG, Jiang X, et al. (2012) U.S. Food and drug administration approval: peginterferon-alfa-2b for the adjuvant treatment of patients with melanoma. Oncologist 17(10):1323-1328. https://doi.org/10.1634/theoncologist.2012-0123.

14. Schuchter LM (2017) Adjuvant melanoma therapy head-spinning progress. N Engl J Med 377(19): 1888-1890. https://doi.org/10.1056/NEJMe171 1199.

15. U.S. Food \& Drug Administration (2019) FDA approves pembrolizumab for adjuvant treatment of melanoma. https://www.fda.gov/drugs/drugapprovals-and-databases/fda-approves-

pembrolizumab-adjuvant-treatment-melanoma. Accessed 20 Nov 2020.

16. Eggermont AMM, Blank CU, Mandala $M$, et al. (2018) Adjuvant pembrolizumab versus placebo in resected stage III melanoma. N Engl J Med 378(19): 1789-1801. https://doi.org/10.1056/NEJMoa180 2357.

17. Study of Pembrolizumab (MK-3475) Versus placebo after complete resection of high-risk stage iii melanoma (MK-3475-054/1325-MG/KEYNOTE-054) . https://clinicaltrials.gov/ct2/show/NCT0236259 4?term $=$ KEYNOTE $+054 \& d r a w=2 \&$ rank $=1$. Accessed 20 Oct 2020 
18. Balch CM, Gershenwald JE, Soong SJ, et al. (2009) Final version of 2009 AJCC melanoma staging and classification. J Clin Oncol 27(36):6199-6206. https://doi.org/10.1200/JCO.2009.23.4799.

19. BCRA, Cotizaciones por fecha- Mercado de Cambios - Cotizaciones cierre vendedor al 27/7/2020 [Internet]. Available from: http://www.bcra.gob.ar/ PublicacionesEstadisticas/Tipos_de_cambios.asp. Accessed 27 July 2020.

20. Adam T, Murray CJL (2003) Making choices in health: WHO guide to cost-effectiveness analysis, vol 1. World Health Organization.

21. Williams C, Lewsey JD, Mackay DF, et al. (2017) Estimation of survival probabilities for use in costeffectiveness analyses: a comparison of a multi-state modeling survival analysis approach with partitioned survival and markov decision-analytic modeling. Med Decis Mak 37(4):427-439. https:// doi.org/10.1177/0272989X16670617.

22. Williams C, Lewsey JD, Briggs AH, et al. (2017) Cost-effectiveness Analysis in R Using a Multi-state Modeling Survival Analysis Framework: A Tutorial. Med Decis Mak 37(4):340-352. https://doi.org/10. 1177/0272989X16651869.

23. NICE. Partitioned survival analysis TSD - NICE Decision Support Unit [Internet]. http://nicedsu. org.uk/technical-support-documents/partitionedsurvival-analysis-tsd/. Accessed 20 Oct 2020.

24. Putter H, Fiocco M, Gekus RB (2007) Tutorial in biostatistics: competing risk and multi-state models. Stat Med 26(11):2389-2430. https://doi.org/10. 1002/sim.2712.

25. Latimer N (2011) NICE DSU technical support document 14: survival analysis for economic evaluations alongside clinical trials-extrapolation with patient-level data report by the decision support unit [Internet]. https://www.nicedsu.org.uk.

26. Flatiron Health (2018) Flatiron database. https:// rwe.flatiron.com.

27. Instituto Nacional de Estadística y Censos (INDEC) (2013) Tablas abreviadas de mortalidad por sexo y edad 2008-2010. Total del país y provincias. Serie Análisis demográfico, no 37.

28. Schachter J, Ribas A, Long GV, et al. (2017) Pembrolizumab versus ipilimumab for advanced melanoma: final overall survival results of a multicentre, randomised, open-label phase 3 study (KEYNOTE006). Lancet 390(10105):1853-1862. https://doi. org/10.1016/S0140-6736(17)31601-X.

29. Robert C, Karaszewska B, Schachter J, et al. (2015) Improved overall survival in melanoma with combined dabrafenib and trametinib. N Engl J Med 372(1):30-39.

NEJMoa1412690.

https://doi.org/10.1056/

30. Robert C, Long GV, Brady B, et al. (2015) Nivolumab in previously untreated melanoma without BRAF mutation. N Engl J Med 372(4):320-330. https://doi.org/10.1056/NEJMoa1412082.

31. Larkin J, Chiarion-Sileni V, Gonzalez R, et al. (2015) Combined nivolumab and ipilimumab or monotherapy in untreated Melanoma. N Engl J Med 373(1):23-34. https://doi.org/10.1056/ NEJMoa1504030.

32. Hodi FS, Chesney J, Pavlick AC, et al. (2016) Combined nivolumab and ipilimumab versus ipilimumab alone in patients with advanced melanoma: 2-year overall survival outcomes in a multicentre, randomised, controlled, phase 2 trial. Lancet Oncol 17(11):1558-1568. https://doi.org/10. 1016/S1470-2045(16)30366-7.

33. Ascierto PA, McArthur GA, Dréno B, et al. (2016) Cobimetinib combined with vemurafenib in advanced BRAFV600-mutant melanoma (coBRIM): updated efficacy results from a randomised, doubleblind, phase 3 trial. Lancet Oncol 17(9):1248-1260. https://doi.org/10.1016/S1470-2045(16)30122-X.

34. McArthur GA, Chapman PB, Robert C, et al. (2014) Safety and efficacy of vemurafenib in BRAFV600E and BRAFV600K mutation-positive melanoma (BRIM-3): Extended follow-up of a phase 3, randomised, open-label study. Lancet Oncol 15(3): 323-332. https://doi.org/10.1016/S14702045(14)70012-9.

35. Long GV, Stroyakovskiy D, Gogas H, et al. (2015) Dabrafenib and trametinib versus dabrafenib and placebo for Val600 BRAF-mutant melanoma: a multicentre, double-blind, phase 3 randomised controlled trial. Lancet 386(9992):444-451. https:// doi.org/10.1016/S0140-6736(15)60898-4.

36. Augustovski FA, Irazola VE, Velazquez AP, et al. (2009) Argentine valuation of the EQ-5D health states. Value Heal 12(4):587-596. https://doi.org/ 10.1111/j.1524-4733.2008.00468.x.

37. Beusterien KM, Szabo SM, Kotapati S, et al. (2009) Societal preference values for advanced melanoma health states in the United Kingdom and Australia. Br J Cancer 101(3):387-389. https://doi.org/10. 1038/sj.bjc. 6605187 .

38. Nomenclators HPGD; CABA; PAMI; IAPOSS; https://www.boletinoficial.gob.ar/detalleAviso/ primera/217123/20190923; https://documento sboletinoficial.buenosaires.gob.ar/publico/PE-RESMSGC-MSGC-577-20-ANX.pdf; http://institucio nal.pami.org.ar/files/boletines_inssjp/RESOL-2019- 
9-INSSJP-SE-INSSJP.pdf (list of values for the relative units); https://prestadores.pami.org.ar/bot nomenclador_unico.php (list of practices with relative units); https://www.santafe.gov.ar/index.php/ web/content/download/252775/1329102/file/ ARANCELES\%20PRACTICAS\%20MEDICAS\% 20DISP\%2078-19.pdf.

39. Coit DG, Thompson JA, Albertini MR, et al. (2019) Cutaneous melanoma, version 2. JNCCN J Natl Compr Cancer Netw 17(4):367-402. https://doi. org/10.6004/jnccn.2019.0018.

40. Seidler AM, Pennie ML, Veledar E, et al. (2010) Economic burden of melanoma in the elderly population: population-based analysis of the surveillance, epidemiology, and end results (SEER)medicare data. Arch Dermatol 146(3):249-256. https://doi.org/10.1001/archdermatol.2009.389.

41. Chastek B, Harley C, Kallich J, et al. (2012) Health care costs for patients with cancer at the end of life. J Oncol Pract. https://doi.org/10.1200/JOP.2011. 000469 .

42. Grupo Alfa Beta. http://www.alfabeta.net/vad/srv. Accessed 27 July 2020.

43. Expert Input and Market research MSD Argentina 2020.

44. Ministerio de Economía, Economic Data, Activity level. GDP per capita in current US\$, 2019 period. https://www.economia.gob.ar/datos/.
45. WHO (2017) WHO-CHOICE methods. WHO. http://www.who.int/choice/cost-effectiveness/ methods/en/.

46. Bensimon AG, Zhou ZY, Jenkins M, et al. (2019) Cost-effectiveness of pembrolizumab for the adjuvant treatment of resected high-risk stage III melanoma in the United States. J Med Econ 22(10): 981-993. https://doi.org/10.1080/13696998.2019. 1609485 .

47. Suciu S, Eggermont AMM, et al. (2018) Relapse-free survival as a surrogate for overall survival in the evaluation of stage ii-iii melanoma adjuvant therapy. J Nat Cancer Ins 110(1):87-96. https://doi.org/ 10.1093/jnci/djx133.

48. Eggermont AMM, Chiarion-Sileni V, Grob JJ, et al. (2019) Adjuvant ipilimumab versus placebo after complete resection of stage III melanoma: long termfollow-up results of the European Organisation for Research and Treatment of Cancer 18071 double-blind phase 3 randomised trial. Eur J Cancer 119:1-10. https://doi.org/10.1016/j.ejca.2019.07. 001.

49. Eggermont AMM, Chiarion-Sileni V, Grob JJ, et al. (2016) Prolonged survival in stage III melanoma with ipilimumab adjuvant therapy. N Engl J Med 375(19):1845-1855. https://doi.org/10.1056/ 